\title{
BMJ open Clinical implementation of a new antibiotic prophylaxis regimen for percutaneous endoscopic gastrostomy
}

\author{
Jesper Lagergren, ${ }^{1,2}$ Fredrik Mattsson, ${ }^{1}$ Pernilla Lagergren ${ }^{1}$
}

To cite: Lagergren J, Mattsson F, Lagergren $P$. Clinical implementation of a new antibiotic prophylaxis regimen for percutaneous endoscopic gastrostomy. BMJ Open 2013;3:e003067. doi:10.1136/bmjopen-2013003067

- Prepublication history and additional material for this paper is available online. To view these files please visit the journal online (http://dx.doi.org/10.1136/ bmjopen-2013-003067)

Received 16 April 2013 Revised 29 April 2013 Accepted 30 April 2013

This final article is available for use under the terms of the Creative Commons Attribution Non-Commercial 2.0 Licence; see http://bmjopen.bmj.com

\footnotetext{
${ }^{1}$ Unit of Upper Gastrointestinal Research, Department of Molecular Medicine and Surgery, Karolinska Institutet, Stockholm, Sweden 2Division of Cancer Studies, King's College London, London, UK
}

\section{Correspondence to} Professor Jesper Lagergren; jesper.lagergren@ki.se

\begin{abstract}
Objectives: This study was undertaken to test the extent to which a new antibiotic prophylaxis regimen for percutaneous endoscopic gastrostomy (PEG), identified as a justified and simpler alternative to conventional regimen in a randomised clinical trial, has been adopted in clinical practice.

Design: A Swedish nationwide implementation survey, conducted in February 2013, assessed the level of clinical implementation of a $20 \mathrm{ml}$ dose of oral solution of sulfamethoxazole and trimethoprim deposited in the PEG catheter immediately after insertion. All hospitals inserting at least five PEGs annually were identified from the Swedish Patient Registry. A clinician involved in the PEG insertions at each hospital participated in a structured telephone interview addressing their routine use of antibiotic prophylaxis.
\end{abstract}

Setting: All Swedish hospitals inserting PEGs $(n=60)$. Participants: Representatives of PEG insertions at each of the 60 eligible hospitals participated $(100 \%$ participation).

Main outcome measures: Use of routine antibiotic prophylaxis for PEG.

Results: A total of $32(53 \%)$ of the 60 hospitals had adopted the new regimen. It was more frequently adopted in university hospitals $(67 \%)$ than in community hospitals (41\%). An annual total of 1813 $(70 \%)$ of 2573 patients received the new regimen. Higher annual hospital volume was associated with a higher level of adoption of the new regimen $(80 \%$ in the highest vs $31 \%$ in the lowest).

Conclusions: The clinical implementation of the new antibiotic prophylaxis regimen for PEG was high and rapid ( $70 \%$ of all patients within 3 years), particularly in large hospitals.

\section{INTRODUCTION}

A main goal of clinical research is to improve clinical practice. Yet, evaluations of the implementation of novel research findings are rare and the methodological quality of the existing literature is limited. ${ }^{1}$ The process from revealing a clinically relevant research finding to having it adopted in the clinical setting is often difficult and requires

\section{ARTICLE SUMMARY}

Article focus

- A main goal of clinical research is to change clinical practice, but evaluations of the clinical implementation of novel research findings are rare.

- A randomised clinical trial suggested a new antibiotic prophylaxis regimen with oral solution deposited in the newly inserted percutaneous endoscopic gastrostomy (PEG) catheter could replace conventional intravenous administration.

\section{Key messages}

- This study provides encouraging evidence of a high $(70 \%$ of all patients) and rapid (within 3 years) clinical implementation of a new antibiotic prophylaxis regimen for PEG, based on the results of a single randomised clinical trial.

- The implementation in routine clinical practice was substantially higher at university hospitals and at hospitals with a higher annual number of PEG insertions compared with smaller hospitals.

Strengths and limitations of this study

- Strengths include the ability to identify all hospitals inserting PEGs in Sweden, the 100\% complete inclusion of all eligible hospitals and the use of structured telephone interviews to collect the data.

- A limitation is the uncertainty whether the results in Sweden are representative of those of other countries.

resources and directed implementation strategies. $^{2-4}$ Many patients do not receive appropriate care or receive unnecessary or harmful treatments. ${ }^{5}$ This study was undertaken to evaluate the potential clinical implementation of a new antibiotic prophylaxis regimen used to reduce infectious complications after the insertion of percutaneous endoscopic gastrostomy (PEG) for enteral nutrition in patients with eating difficulties, as recommended in a randomised clinical trial published in $2010 .{ }^{6}$ The new regimen was a $20 \mathrm{ml}$ dose of the oral solution of sulfamethoxazole and trimethoprim deposited in 
the PEG catheter immediately after its insertion. This regimen was tested against a standard of an intravenous dose of $1.5 \mathrm{~g}$ cefuroxime administered $1 \mathrm{~h}$ before inserting PEG. The trial included 234 patients (116 with the new regimen and 118 with standard regimen), and all patients and evaluators were blinded to which prophylaxis was used. The study outcome was clinical signs of infection, supported by bacterial cultures and blood tests. The trial was a non-inferiority study, that is, if the new regimen was at least as effective as standard treatment it would be preferable since it had advantages that favoured its use, that is, easier to administer, cheaper, not given unnecessarily to the $10 \%$ of the cases where PEG is not possible to insert. ${ }^{7} 8$ The trial showed that the new regimen was at least as effective in preventing infections, and the results were published as an original article in $B M J .{ }^{6}$ We hypothesised that the trial had changed clinical practice and tested this hypothesis in a nationwide Swedish survey.

\section{METHODS}

Presentation of the trial results

The trial that was assessed in this study was published in $B M J$ in $2010 .{ }^{6}$ Moreover, a summary of the study was published in the Swedish Medical Journal (Lakartidningen). An oral presentation of the trial was conducted at a National Swedish conference in surgery. Finally, the study was presented at a Swedish meeting for clinicians with an interest in PEG.

\section{Study design and setting}

This was a population-based, nationwide Swedish implementation survey. All hospitals that had conducted an average of at least five PEG insertions/year during the 3-year period 2009-2011 were identified from the Swedish Patient Registry. This registry is complete nationwide and collects data of all diagnoses and surgical procedures (including endoscopic procedures) during in-hospitalisations or specialist outpatient care, and has been found to have excellent completeness and accurateness. ${ }^{9}$ A project nurse contacted the clinician responsible for the PEG activities at each of the identified hospitals and conducted a structured telephone interview strictly according to a study questionnaire. There was no mention about the trial under study until after the questions about current antibiotic prophylaxis were completed. All interviews were completed during the period from 30 January to 28 February 2013. The fact that the test regimen was entirely new facilitated the assessment of its potential implication in the clinical setting. The study was approved by the Regional Ethical Review Board in Stockholm, Sweden.

\section{Statistical analyses}

The hospitals were categorised into three groups: (1) university hospitals, (2) county hospitals or (3) community hospitals. We also estimated the number of inserted PEGs for 2012. This was performed by calculating the arithmetic mean of the number of inserted PEGs during the 3-year period 2009-2011 according to the Swedish Patient Registry for each hospital, and then summarised for all patients. Furthermore, we stratified all patients in 2012 by the use of antibiotic regimen, and in a similar way stratified each category of hospital. An additional categorisation of the hospitals into quintiles, based on the ranking of each hospital according to the estimated mean number of patients, was also conducted. The data management and the diagrams were conducted using the SAS (V.9.2; SAS Institute Inc, Cary, North Carolina, USA).

\section{RESULTS}

There were 60 eligible hospitals in Sweden that inserted at least 5 PEGs/year. All these hospitals participated in the interviews (100\% participation rate). A total of 17 (28\%) hospitals did not routinely use any antibiotic prophylaxis, most being community hospitals (71\%). The rates of implementation of the new regimen at all hospitals and at the three categories of hospitals are presented in table 1 . The total number of hospitals which had implemented the new regimen was $32(53 \%)$; among hospitals using antibiotic prophylaxis, this rate was 32 out of $43(74 \%)$. The new regimen was most frequently adopted in university hospitals $(67 \%)$, while it was less commonly adopted in community hospitals $(41 \%)$. Of all hospitals that had introduced the new regimen, $25(78 \%)$ had adopted the new regimen within the year 2011. Reasons for having changed to the new regimen are presented in table 1 . The most common reason was having read the article published in BMJ $(63 \%)$.

Translated to the number of patients, an annual total of $1813(70 \%)$ of 2573 patients received the new regimen (figure 1, table 2). Excluding hospitals not using antibiotic prophylaxis, the frequency using the new regimen was $79 \%$. A higher annual hospital volume was associated with an increased adoption of the new regimen; the frequency of use of the new regimen was $31 \%$ in the lowest quintile of hospitals and $80 \%$ in the highest (table 2). The distribution of antibiotic prophylaxis according to the number of patients treated at the three categories of hospitals showed a higher frequency of the new regimen in university hospitals $(84 \%)$, followed by county hospitals $(71 \%)$ and community hospitals (45\%; figure 2).

\section{DISCUSSION}

This study shows that the new antibiotic prophylaxis, with a solution of sulfamethoxazole and trimethoprim given directly in the newly inserted PEG, had replaced standard prophylaxis with intravenously given antibiotics to a considerable extent in Sweden.

Methodological strengths of the study include the ability to identify all hospitals inserting PEGs in Sweden 
Table 1 Use of antibiotic regimen as part of the insertion of percutaneous endoscopic gastrostomy in Sweden, presented as number of hospitals and hospitals divided by the three hospital categories community hospitals, county hospitals and university hospitals

\begin{tabular}{|c|c|c|c|c|c|c|c|c|}
\hline & \multirow{2}{*}{\multicolumn{2}{|c|}{$\begin{array}{l}\text { All hospitals } \\
(n=60)\end{array}$}} & \multicolumn{6}{|c|}{ Hospital category } \\
\hline & & & \multicolumn{2}{|c|}{ Community $(\mathrm{n}=27)$} & \multicolumn{2}{|c|}{ County $(n=24)$} & \multicolumn{2}{|c|}{ University $(n=9)$} \\
\hline & Number & Per cent & Number & Per cent & Number & Per cent & Number & Per cent \\
\hline \multicolumn{9}{|c|}{ Antibiotic prophylaxis regimen } \\
\hline $\mathrm{New}^{*}$ & 32 & 53 & 11 & 41 & 15 & 63 & 6 & 67 \\
\hline Other† & 11 & 18 & 4 & 15 & 5 & 21 & 2 & 22 \\
\hline None & 17 & 28 & 12 & 44 & 4 & 17 & 1 & 11 \\
\hline \multicolumn{9}{|l|}{ Knowledge of the trial } \\
\hline Yes & 38 & 63 & 12 & 44 & 18 & 75 & 8 & 89 \\
\hline No & 22 & 37 & 15 & 56 & 6 & 25 & 1 & 11 \\
\hline \multicolumn{9}{|c|}{ Hospitals using the new regimen } \\
\hline \multicolumn{9}{|c|}{ Year of introduction } \\
\hline 2010 & 10 & 31 & 2 & 7 & 7 & 29 & 1 & 11 \\
\hline 2011 & 15 & 47 & 4 & 15 & 7 & 29 & 4 & 44 \\
\hline 2012 & 7 & 22 & 5 & 19 & 1 & 4 & 1 & 11 \\
\hline \multicolumn{9}{|c|}{ Reason for introduction } \\
\hline Read the article & 20 & 63 & 5 & 45 & 9 & 60 & 6 & 100 \\
\hline Scientific meeting & 7 & 22 & 3 & 27 & 4 & 27 & 0 & 0 \\
\hline Colleague & 4 & 13 & 2 & 18 & 2 & 13 & 0 & 0 \\
\hline Unknown & 1 & 3 & 1 & 9 & 0 & 0 & 0 & 0 \\
\hline
\end{tabular}

*20 $\mathrm{ml}$ dose of oral solution of sulfamethoxazole and trimethoprim deposited in the PEG catheter immediately after insertion. †ntravenously administered antibiotics before percutaneous endoscopic gastrostomy insertion.

through the Patient Registry, making it population-based in design and reducing the risk of selection bias. The $100 \%$ complete inclusion of all eligible hospitals further counteracted biased selection. Finally, the use of structured telephone interviews to collect the data should minimise the misclassification of the information collected. These advantages should provide high internal validity of the study. ${ }^{1}$ The external validity, however, is

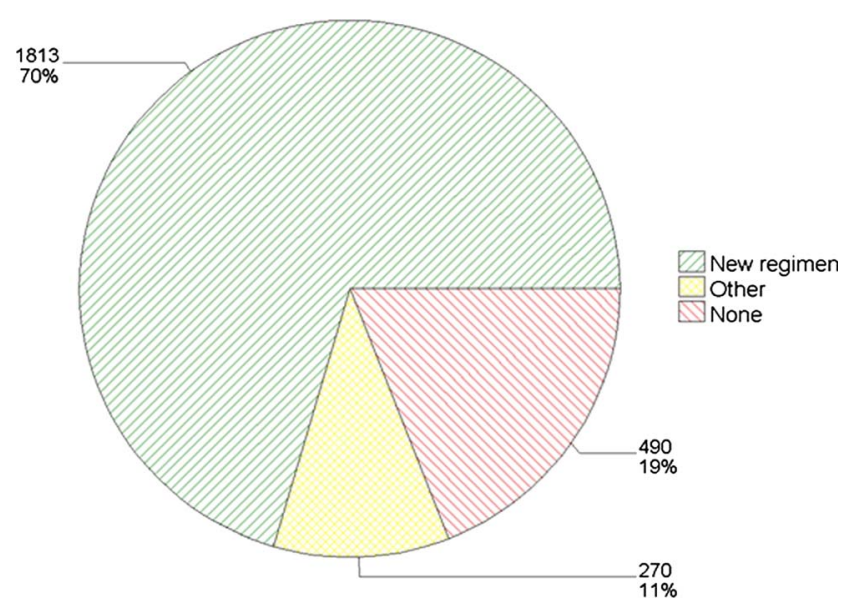

Figure 1 Use of antibiotic regimen as part of the insertion of percutaneous endoscopic gastrostomy in Sweden, presented as number of patients. New regimen: $20 \mathrm{ml}$ dose of oral solution of sulfamethoxazole and trimethoprim deposited in the percutaneous endoscopic gastrostomy (PEG) catheter immediately after insertion. Other regimen: intravenously administered antibiotics before PEG insertion. uncertain since it is unknown whether the results in Sweden are representative of those of other countries. The trial being followed up was conducted in Sweden, which should facilitate its implementation in this country in particular, while the implementation in other countries might be more limited.

Scientific evidence suggests that enabling research findings to change clinical practice requires comprehensive approaches and effective strategies at different levels, tailored to specific settings and target groups. ${ }^{3-5}$ The present study shows that it is possible to influence clinical practice based on a single randomised clinical trial if the results are convincing and easy to adopt in routine care. The implementation of the new regimen tested in the present study was facilitated by the fact that it had practical advantages and was cheaper to use compared with the standard regimen. In other trials, the tested treatment might often be more complex to use and more costly, which might make its introduction into routine clinical use less likely. For example, another implementation study of an endoscopic procedure was a large cohort study in the USA which showed that low rates of duodenal biopsy remained in more recent calendar periods in patients with clinical indications for biopsy to identify coeliac disease despite clinical recommendations to conduct such biopsies more frequently. ${ }^{10}$

University hospitals and PEG high-volume hospitals were clearly more likely to adopt the new regimen compared with smaller hospitals. This might be due to a higher academic interest and a higher interest in the PEG procedure, respectively, which might increase the 
Table 2 Use of antibiotic regimen as part of the insertion of percutaneous endoscopic gastrostomy in Sweden, presented as number of patients per year and hospital, divided in quintiles of hospital volume (12 hospitals in each quintile)

\begin{tabular}{|c|c|c|c|c|c|c|c|c|c|c|c|c|}
\hline \multirow{3}{*}{$\begin{array}{l}\text { Antibiotic } \\
\text { prophylaxis } \\
\text { regimen }\end{array}$} & \multirow{2}{*}{\multicolumn{2}{|c|}{$\frac{\text { All }}{(n=2573)}$}} & \multicolumn{10}{|c|}{ Quintile } \\
\hline & & & \multicolumn{2}{|c|}{$1(n=101)$} & \multicolumn{2}{|c|}{$2(n=220)$} & \multicolumn{2}{|c|}{$3(n=341)$} & \multicolumn{2}{|c|}{$4(n=495)$} & \multicolumn{2}{|c|}{$5(n=1417)$} \\
\hline & $\mathbf{N}$ & Per cent & $\overline{\mathbf{N}}$ & Per cent & $\overline{\mathbf{N}}$ & Per cent & $\overline{\mathbf{N}}$ & Per cent & $\overline{\mathbf{N}}$ & Per cent & $\mathbf{N}$ & Per cent \\
\hline $\mathrm{New}^{\star}$ & 1813 & 70 & 31 & 31 & 115 & 52 & 202 & 59 & 335 & 68 & 1130 & 80 \\
\hline Othert & 270 & 11 & 20 & 20 & 60 & 27 & 114 & 33 & 77 & 15 & 0 & 0 \\
\hline None & 490 & 19 & 50 & 49 & 45 & 20 & 25 & 7 & 83 & 17 & 287 & 20 \\
\hline
\end{tabular}

*20 ml dose of oral solution of sulfamethoxazole and trimethoprim deposited in the PEG catheter immediately after insertion.

†Intravenously administered antibiotics before PEG insertion.

PEG, percutaneous endoscopic gastrostomy.

likelihood of reading scientific articles and willingness to adopt new treatments. The lack of use of any antibiotic prophylaxis was not negligible, which might not be entirely justified considering the amount of evidence showing a high risk of infectious complications, ${ }^{11}$ and an absolute risk reduction of such complications by 14$17 \%$ with the use of antibiotic prophylaxis. ${ }^{78}$

Although it is pleasing to find such rapid and positive uptake of an innovation, any recommendation for uptake of this new practice should be made with caution since it is based on one clinical trial of 200 patients only. A systematic review, which incorporates multiple sources of evidence, might provide a stronger basis for changing clinical practice.

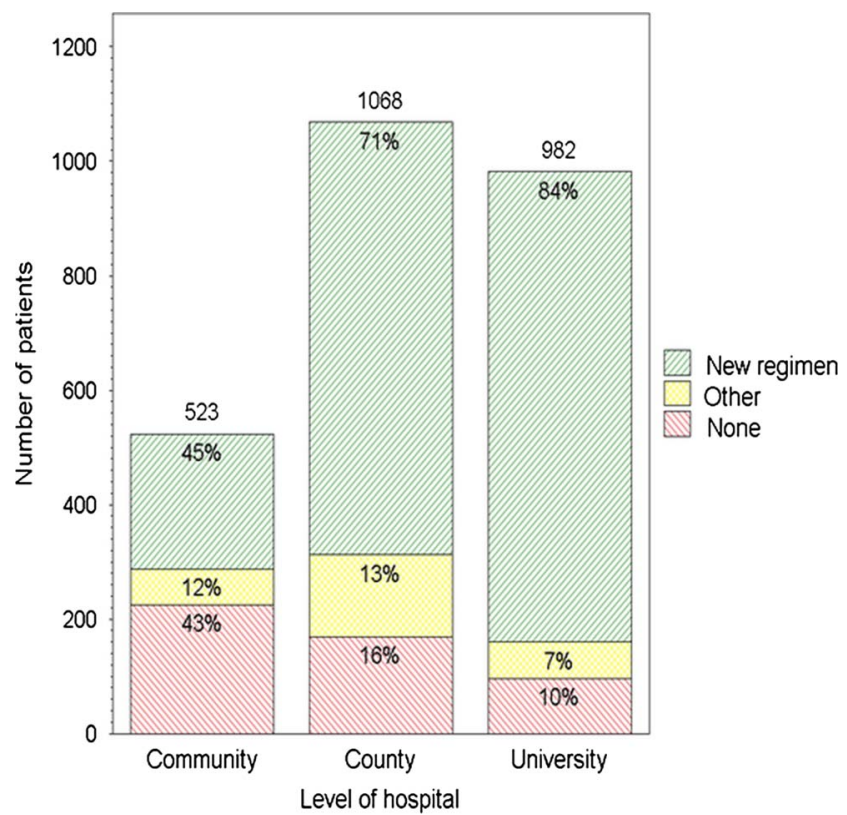

Figure 2 Use of antibiotic regimen as part of the insertion of percutaneous endoscopic gastrostomy (PEG) in Sweden, presented as number of patients, and divided by the three hospital categories community hospitals, county hospitals and university hospitals. New regimen: $20 \mathrm{ml}$ dose of oral solution of sulfamethoxazole and trimethoprim deposited in the PEG catheter immediately after insertion. Other: intravenously administered antibiotics before PEG insertion.
In conclusion, this nationwide complete Swedish survey provides encouraging evidence of a high $(53 \%$ of all hospitals and $70 \%$ of all patients) and rapid clinical implementation of a new antibiotic prophylaxis regimen for PEG based on the results of one well-designed randomised clinical trial. The level of adoption of the new regimen was substantially higher in university hospitals and PEG high-volume hospitals compared with smaller hospitals. This should encourage further randomised clinical trials addressing clinically relevant topics.

Acknowledgements We are grateful to the research nurse Isabella Linder Rosén for conducting all interviews.

Contributors $\mathrm{JL}$ had the original idea to conduct this study. JL and PL planned the study and completed the data collection. FM did the analyses, tables and figures. JL made the first draft of the article, and PL and FM contributed with input on the article writing. $\mathrm{JL}$ is responsible for the overall content as guarantor. All authors have read and approved the final manuscript.

Funding The Swedish Research Council (SIMSAM) and through the regional agreement on medical training and clinical research (ALF) between Stockholm County Council and Karolinska Institutet.

Competing interests None.

Ethics approval The study was approved by the Regional Ethical Review Board in Stockholm, Sweden (diary number 2013/131-32).

Provenance and peer review Not commissioned; externally peer reviewed.

Data sharing statement Data sharing: full dataset and statistical code are available from the corresponding author at jesper.lagergren@ki.se.

STROBE guidelines We followed the recommendation of the STROBE guidelines for observational studies.

\section{REFERENCES}

1. Fan $\mathrm{E}$, Laupacis $\mathrm{A}$, Pronovost $\mathrm{PJ}$, et al. How to use an article about quality improvement. JAMA 2010;304:2279-87.

2. Gilbody S, Whitty P, Grimshaw J, et al. Educational and organizational interventions to improve the management of depression in primary care: a systematic review. JAMA 2003;289:3145-51.

3. Althabe $F$, Buekens $P$, Bergel $E$, et al. A behavioral intervention to improve obstetrical care. N Engl J Med 2008;358:1929-40.

4. Bessen T, Clark R, Shakib S, et al. A multifaceted strategy for implementation of the Ottawa ankle rules in two emergency departments. BMJ 2009;339:b3056.

5. Grol R, Grimshaw J. From best evidence to best practice: effective implementation of change in patients' care. Lancet 2003;362:1225-30. 
6. Blomberg J, Lagergren P, Martin L, et al. Novel approach to antibiotic prophylaxis in percutaneous endoscopic gastrostomy (PEG): randomised controlled trial. BMJ 2010;341:c3115.

7. Lipp A, Lusardi G. Systemic antimicrobial prophylaxis for percutaneous endoscopic gastrostomy. Cochrane Database Syst Rev (Online) 2006;(4):CD005571.

8. Lipp A, Lusardi G. A systematic review of prophylactic antimicrobials in PEG placement. J Clin Nurs 2009;18:938-48.
9. Ludvigsson JF, Andersson E, Ekbom A, et al. External review and validation of the Swedish national inpatient register. BMC Public Health 2011;11:450.

10. Lebwohl B, Tennyson CA, Holub JL, et al. Sex and racial disparities in duodenal biopsy to evaluate for celiac disease. Gastrointest Endosc 2012;76:779-85.

11. Blomberg J, Lagergren J, Martin L, et al. Complications after percutaneous endoscopic gastrostomy in a prospective study. Scand J Gastroenterol 2012;47:737-42. 